\title{
Uptrend in distress and psychiatric symptomatology in pregnant women during the COVID-19 pandemic
}

Nicolas Berthelot (Ph.D.), ${ }^{1,4,5,6}$ Roxanne Lemieux (Ph.D.), ${ }^{1,4}$ Julia Garon-Bissonnette

(B.A.), ${ }^{2,4,5,6}$ Christine Drouin-Maziade (Psy.D.), ${ }^{1,4}$ Élodie Martel (B.A.), ${ }^{2,4,5}$ \& Michel Maziade (M.D.) $)^{3,5}$

\section{Affiliations}

1. Department of Nursing Sciences, Université du Québec à Trois-Rivières, TroisRivières (Quebec), Canada

2. Department of Psychology, Université du Québec à Trois-Rivières, Trois-Rivières (Quebec), Canada

3. Faculty of Medicine, Université Laval, Quebec city (Quebec), Canada

4. Centre d'études interdisciplinaires sur le développement de l'enfant et la famille, Trois-Rivières (Quebec), Canada

5. CERVO Brain research center, Quebec city (Quebec), Canada

6. Interdisciplinary research center on intimate relationship problems and sexual abuse, Montreal (Quebec), Canada

\section{Corresponding author}

Nicolas Berthelot, Ph.D.

Professor of psychology

Université du Québec à Trois-Rivières, Department of Nursing sciences

Trois-Rivières, Québec, Canada

3351 boul. des Forges, PO Box 500

Nicolas.berthelot@uqtr.ca

Tel. : 1-819-376-5011, ext. 3487

Wordcount: 2993 words

Date of the submission: 2020-05-18 
Conflict of interest: The authors report no conflict of interest

Funding: No specific funding was received for this study 


\begin{abstract}
Introduction: Prenatal maternal distress has a negative impact on the course of pregnancy, fetal development, offspring development and later psychopathologies. The study aimed to determine the extent to which the Coronavirus disease 2019 (COVID-19) pandemic may aggravate pregnant women prenatal distress and psychiatric symptomatology.
\end{abstract}

Material and methods: Two cohorts of pregnant volunteer women were evaluated, one that was recruited before the COVID-19 pandemic $(n=496)$ through advertisements in prenatal clinics in Quebec, Canada, from April 2018 to March 2020; the other $(n=1258)$ was recruited online during the pandemic from April 2 to April 13 2020. Prenatal distress and psychiatric symptomatology were measured with the Kessler Distress Scale (K10), Post-traumatic Checklist for DSM-5 (PCL-5), Dissociative Experiences Scale (DES-II) and Positive and Negative Affect Schedule (PANAS).

Results: The 1754 pregnant women $\left(\mathrm{M}_{\mathrm{age}}=29.27, \mathrm{SD}=4.23\right)$ were between 4 and 41 gestational weeks $(\mathrm{M}=24.80, \mathrm{SD}=9.42)$, were generally educated $(91.3 \%$ had post-high school training) and financially well-resourced $(85.3 \%$ were above the low-income cutoff). A multivariate analysis of covariance controlling for age, gestational age, household income, education and lifetime psychiatric disorders showed a large effect size in the difference between the two cohorts on psychiatric symptoms (Wilks' $\lambda=0.68$, $F_{6,1400}=108.50, p<0.001$, partial $\eta^{2}=0.32$ ). According to post-hoc analyses of covariance, the COVID-19 women reported higher levels of depressive and anxiety symptoms $(E S=0.57)$, dissociative symptoms $(E S=0.22$ and 0.25$)$, symptoms of posttraumatic stress disorder $(\mathrm{ES}=0.19)$, negative affectivity $(\mathrm{ES}=0.96)$ and less positive affectivity $(\mathrm{ES}=0.95)$ than the pre-COVID-19 cohort. Women from the COVID-19 cohort were more likely than pre-COVID-19 women to present clinically significant levels of depressive and anxiety symptoms $[\mathrm{OR}=1.94, \chi 2(1)=10.05, p=.002]$. Multiple regression analyses indicated that COVID-19 pregnant women having a previous psychiatric diagnosis or low income would be more prone to elevated distress and psychiatric symptoms.

Conclusions: Pregnant women assessed during the COVID-19 pandemic reported more distress and psychiatric symptoms than pregnant women assessed before the pandemic, 
mainly in the form of depression and anxiety symptoms. Given the harmful consequences of prenatal distress on mothers and offspring, the presently observed upsurge of symptoms in pregnant women calls for special means of clinical surveillance.

Keywords: COVID-19, anxiety, depression, PTSD, mental health, prenatal, pregnancy, mothers

Abbreviations: PTSD, post-traumatic stress disorder; ADHD, Attention-deficit hyperactivity disorder; ADD, Attention deficit disorder; CND, Canadian dollar Key message: The COVID-19 pandemic is associated with an uptrend in psychological distress and psychiatric symptomatology in pregnant women. This may have a negative impact on the course of pregnancy, fetal development and offspring development. 


\section{Introduction}

There is robust evidence that prenatal psychological distress is harmful to the mother, the fetus and eventually the child. Indeed, maternal stress during pregnancy has been associated with serious negative outcomes including poor maternal psychosocial functioning, ${ }^{1}$ parenting difficulties, ${ }^{1}$ lower infant birth weight,${ }^{2}$ earlier infant gestational age ${ }^{2}$ offspring psychopathology, ${ }^{3}$ alterations in brain development, ${ }^{4}$ and poorer socioemotional ${ }^{5}$ and cognitive development. ${ }^{6}$ The detrimental effects of prenatal maternal psychological distress would not be limited to the most vulnerable populations since higher levels of anxiety and depression during pregnancy would be linked to alterations in fetal and infant brain development even in women with low-risk pregnancies, high levels of education and high socioeconomic status. ${ }^{4,7}$

While maternal prenatal psychological distress is common in community samples of pregnant women, ${ }^{4}$ major stressors such as health crises and natural disasters would increase prenatal stress and exert long-lasting effects on child development. ${ }^{8}$ Coronavirus disease 2019 (COVID-19) has affected everyone around the world while often disrupting medical practices in non COVID-19 cases and specifically in prenatal care. ${ }^{9,} 10$

Understandably, perinatal research on COVID-19 has primarily focused on maternal and neonatal outcomes of COVID-19 infection during pregnancy. The impact of the COVID-19 pandemic on mental health in pregnant women remains unknown. ${ }^{10}$ Similarly, though medical associations worldwide had to provide clinical guidelines on the clinical management of pregnant women and their newborn during the pandemic, recommendations related to maternal mental health were limited by the absence of empirical data. ${ }^{10}$

The aim of the present study was to provide such data. We evaluated to what extent the COVID-19 pandemic provoked an increase of prenatal psychological distress in pregnant women, as measured by two domains of symptoms: i) depressive and anxiety symptoms (i.e. high prenatal distress, high negative affectivity, low positive affectivity) and trauma-related symptoms (i.e. dissociation and symptoms of post-traumatic stress disorder). We also assessed whether a particular subgroup of pregnant women would express more vulnerability. 


\section{Material and methods}

\section{Study Design and sample}

A first cohort of pregnant women was recruited into a longitudinal study in prenatal clinics before the COVID-19 pandemic, from April 2018 to March 1 2020, through an invitation to participate in a research on early life experiences and parenthood. A second cohort was recruited during the active COVID-19 pandemic from April 2 to April 132020 through social media (Facebook and Instagram) and advertisements in prenatal clinics. All participants were volunteers from the Province of Quebec, Canada, where a public health emergency was declared in reaction to COVID-19 on March 13 2020. This culminated into the closure of all non-essential businesses, self-isolation and restriction of non-essential displacements from March 24 until May 4. The enrolment of the COVID-19 pandemic cohort actually took place when the pandemic was well under way. Inclusion criteria were being 18 or older, having sufficient reading skills to complete self-report instruments and being pregnant at the time of assessment. Measures were completed online on a secure portal. Informed consent was obtained from all participants.

\section{Measures}

Two instruments showing strong correlations with anxiety disorders and depression and two questionnaires of trauma-related disorders (post-traumatic stress disorder and dissociation) were selected for measuring prenatal psychological distress. Anxiety and depression were first measured using the 10-item Kessler Psychological Distress Scale (K10). ${ }^{11,12}$ Higher scores at the K10 indicate more symptoms and distress and $76.3 \%$ of respondents with scores $\geq 30$ would meet the criteria for a DSM-IV anxiety, affective or substance use disorder during a diagnostic interview. ${ }^{13}$ Both the English and French versions have similarly satisfactory psychometric properties ${ }^{11}$ and the instrument has been shown to screen adequately for mood and anxiety disorders in pregnant women. ${ }^{14}$ Cronbach's alpha for the K-10 in this study was .88 .

Positive and negative affect were assessed using the Positive and Negative Affect Schedule (PANAS). ${ }^{15,16}$ The Positive Affect scale probes for feelings such as enthusiasm, energy and dynamism whereas the Negative Affect scale catches feelings of anger, fear, 
guilt and nervousness. Scores on the Positive Affect Scale were reversed to reflect low positive affectivity. The original and the French version have shown up as valid and reliable, ${ }^{16,17}$ and PANAS scores were shown to be associated with DSM-IV major depressive disorder in pregnant women. ${ }^{18}$ The Cronbach's alpha for the Positive affect and Negative affect subscales were .85 and .89 respectively.

The Post-traumatic stress disorder (PTSD) Checklist for DSM-5 (PCL-5) assessed post-traumatic stress symptoms during the past month and is based on the PTSD diagnostic criteria of the DSM-5. ${ }^{19,20}$ Its 20 items can be regrouped into four subscales corresponding to the clusters of symptoms of a PTSD diagnosis in the DSM-5: intrusion (Cluster B; 5 items), avoidance of trauma-related stimuli (Cluster C; 2 items), negative alterations in cognitions and mood (Cluster D; 7 items), and alterations in arousal and reactivity (Cluster E; 6 items). Higher scores indicate more severe symptoms (clinical cut-off $\geq 33$ ). ${ }^{20}$ The instrument was previously used in samples of pregnant women ${ }^{21}$ and both the French and the original versions have equally adequate validity and reliability. ${ }^{19}$ The Cronbach's alpha for the PCL-5 in this study was .92.

Dissociation was measured with the depersonalization/derealization (6 items) and absorption/imaginative involvement (9 items) subscales of the Dissociative Experiences

Scale (DES-II). ${ }^{22,23}$ The instrument was previously used in samples of pregnant women ${ }^{24}$ and has good validity and reliability. ${ }^{22,23}$ The Cronbach's alpha for the DES in this study was of .75 for the depersonalization subscale and .83 for the absorption subscale.

A sociodemographic questionnaire was administered to assess potentially confounding variables, including age, gestational age, education, annual income and history of psychiatric disorders. For the latter, participants had to confirm all mental health disorders among the following for which they received a medical diagnosis at one point in their life: major depressive disorder, anxiety disorder, PTSD, eating disorder, bipolar disorder, psychotic disorder, personality disorder, ADHD, or other disorder (Table 1).

\section{Statistical analysis}

Analyses were performed with SPSS, version 24.0. The characteristics of the participants across the two cohorts were compared using t-test for continuous variables 
and chi-squared test for categorical variables. To meet our first objective, we performed a multivariate analysis of covariance (MANCOVA) to evaluate whether the pre-COVID-19 and COVID-19 pandemic cohorts differed on the six symptoms. Subsequent ANCOVAs were performed to examine group differences on the six symptoms separately. Scores were converted to z scores. All analyses controlled for age, gestational age, education level, household income and lifetime psychiatric disorders. We calculated effect sizes (ES) using the difference of adjusted means between the COVID-19 and the pre-COVID19 cohorts divided by a pooled standard deviation. In complement to analyses on continuous variables, we wanted to compare the two cohorts using categorical analyses via established cut-offs for the K10 and PCL-5 and used chi-squared tests to test homogeneity.

To meet our second objective, i.e. identifying a subgroup potentially more vulnerable to prenatal distress during the pandemic, we first conducted in the COVID-19 cohort a factor analysis with Varimax rotation on the six symptoms, in order to diminish the number of meaningful symptoms to be analyzed (see Results and Table S4). A strong factorial structure was obtained. We then performed multiple regressions with the COVID-19 women's characteristics as predictors and their factor score on each of the three factors. Given that three regression analyses were performed, we conservatively applied Bonferroni corrections and the significance level was fixed at $p<.02$.

\section{Ethical approval}

This study was approved by the Institutional Review Board of Université du Québec à Trois-Rivières (\#CER-20-266-10.10) on April 22020.

\section{Results}

The sample consisted of 1754 pregnant women, ranging from 18 to 46 years old $(M=29.27, S D=4.23)$. The characteristics of the two cohorts are presented in Table 1. Among the participants, $91.3 \%$ had post-high school training and $85.3 \%$ were above the low-income cut-off of 33 863CND for a family with two children. The median household income in our sample was between 85000 and 95 000CND. Our sample of pregnant women would thus be highly representative of Quebec's population of mothers since 
$91,5 \%$ of women between 25 and 34 have post-high-school education and the median income for a household of two people or more is of 87 400CND in Quebec. The majority of the sample was Caucasian (95\%). The pre-COVID-19 and COVID-19 cohorts had similar age and lifetime diagnoses of psychiatric disorders, but the COVID-19 cohort was significantly more educated, had higher household income and had slightly older gestational age (Table 1). Of the 1754 participants, 342 had missing data on at least one instrument (Table S1). No demographic difference was found between participants with complete data and those with missing data (Table S2). Moreover, the analyses including only participants with complete data $(n=1412$; Table S3) yielded the same results as the analyses in the total sample $(\mathrm{n}=1754)$ shown below.

Pregnant women from the COVID-19 pandemic cohort reported more prenatal distress than pregnant women from the pre-COVID-19 cohort (MANCOVA; Wilks' $\lambda=0.68, F_{6,1400}=108.50, p<0.001$, partial $\eta^{2}=0.32$ ) even when controlling for the effect of age (Wilks' $\lambda=0.98, F_{6,1400}=4.13, p<0.001$, partial $\eta^{2}=0.02$ ), gestational age (Wilks' $\lambda=0.99, F_{6,1400}=1.26, p=0.28$ ), education (Wilks' $\lambda=0.98, F_{6,1400}=5.16, p<0.001$, partial $\eta^{2}=0.02$ ), household income (Wilks' $\lambda=0.97, F_{6,1400}=8.42, p<0.001$, partial $\eta^{2}=0.04$ ) and lifetime psychiatric diagnoses (Wilks' $\lambda=0.92, F_{6,1400}=21.16, p<0.001$, partial $\eta^{2}=$ 0.08). Post hoc ANCOVAs (Table 2) revealed more severe symptoms of depression and anxiety, higher levels of negative affectivity and lower levels of positive affectivity in the COVID-19 cohort. Pregnant women assessed during the pandemic also reported more symptoms of PTSD and dissociation than women from the pre-COVID-19 cohort. Since a diagnosis of PTSD requires the combination of four types of symptoms (see Methods), we next analyzed PTSD clusters of symptoms separately. We observed that the COVID19 cohort reported more alterations in cognitions and mood (criterion D of a PTSD diagnosis; $F_{1,1563}=28.50, p<0.001, \mathrm{ES}=.29$ ) and alterations in arousal and reactivity (criterion $\mathrm{E} ; F_{1,1562}=20.83, p<0.001, \mathrm{ES}=0.24$ ) but had similar levels of intrusion and avoidance than the pre-COVID-19 cohort.

We then performed categorical analyses of symptoms dividing participants into low/moderate vs high risk of having a DSM disorder based on previously validated K10 clinical cut-off (K10 scores $\geq 30$ ). ${ }^{13}$ Results showed that $10.9 \%$ of the COVID-19 cohort 
had clinically significant symptoms of anxiety and depression in comparison to only $6.0 \%$ of the pre-COVID-19 cohort $[\mathrm{OR}=1.94, \chi 2(1)=10.05, p=.002]$. However, the two cohorts were similar in PTSD symptoms $[\chi 2(1)=1.77, p=.18]$.

The factor analysis of the 6 psychiatric symptoms yielded in the COVID-19 cohort a strong three factors structure explaining respectively $49 \%, 18 \%$ and $14 \%$ of the total variance: Factor 1 was composed of mood and anxiety symptoms (anxiety/depression; negative affectivity; PTSD), Factor 2 of dissociative symptoms and Factor 3 of low positive affectivity (see Table S4). Multiple regression analyses having each a Factor as the predicted variable (Table 3) showed that lifetime psychiatric disorders and household income were the best predictors of Factor $1 \operatorname{mood}$ and anxiety, and of Factor 2 dissociative symptoms. Factor 3 low positive affectivity was predicted by lifetime psychiatric disorders, education levels and gestational age.

\section{Discussion}

To the best of our knowledge, this is the first study reporting a significant surge of distress and psychiatric symptoms in pregnant women during the COVID-19 pandemic. ${ }^{10}$ We contrasted two large cohorts of pregnant women from the general population, one assessed before and the other during the COVID-19 pandemic, by using the same measurements. The public health and mental health relevance of our study is markedly supported by the documented negative impact of psychological distress during pregnancy on both mothers' and offspring's health. ${ }^{1,3-5}$

Our study indeed revealed differences with small to large effect sizes between the pre-COVID-19 and the COVID-19 cohorts on all psychiatric symptoms. More specifically, during the pandemic, pregnant women would mainly exhibit an upsurge of depressive and anxiety symptoms (defined as high psychological distress, high negative affectivity and low positive affectivity) along with some symptoms of dissociation and post-traumatic stress disorder. However, post-hoc analyses revealed that only the clusters of PTSD symptoms overlapping with anxiety and depression (i.e. negative mood and cognitions; alterations in reactivity and arousal), and not symptoms that are more specific to PTSD (i.e. intrusion symptoms and avoidance of trauma-related stimuli), increased during the COVID-19 pandemic. Remarkably, also, a history of a psychiatric disorder 
prior to the pandemic, younger age, lower household income and lower education were all associated with higher maternal psychological distress. It is noteworthy that the differences in symptomatology between the two cohorts remained significant after controlling for the above variables. This finding adds to previous results suggesting that high levels of prenatal distress would not be limited to the most vulnerable subgroups of pregnant women. ${ }^{4,7}$

Moreover, our using a categorical validated cut-off on the symptoms scales to compare the pregnant women with higher symptomatology to those with minimal or lower symptomatology yielded congruent results on changes in psychiatric clinical status. The COVID-19 pregnant women would show up as almost twice more likely to present a level of symptoms meeting a DSM diagnosis of affective, anxiety or substance use disorder, but showed no risk difference for a DSM diagnosis of PTSD which was somewhat congruent with the previous results from the continuous analysis on PTSD clusters of symptoms.

Our findings have clinical meaning for primary and specialized care. Indeed, easily measurable attributes in the clinic, such as a personal history of psychiatric disorder and lower sociodemographic status, would predict higher psychiatric symptomatology in the pandemic and thus definitely higher health risk for the mother and child. ${ }^{1-7}$ As detailed below in the conclusion, our data advocate for special forms of clinical surveillance of pregnant women who exhibit these characteristics, as well as targeted information in primary care facilities and from public informants.

Our findings have also implications for developmental psychopathology. Prenatal psychological distress has been repetitively shown to be associated with poor developmental outcomes in offspring such as alterations in brain development ${ }^{4,7}$ and poorer socioemotional and cognitive development. ${ }^{5,6}$ In that respect, it may be relevant to attempt a comparison of our present findings with previous studies from the same population. For instance, previous Quebec studies have shown that prenatal maternal distress resulting from another stressful event at the population level (the 1998 Quebec Ice Storm; see SDiscussion in the Supplement) had an effect on offspring DNA methylation, ${ }^{25}$ metabolic pathways involved in energy metabolism and protein biosynthesis, ${ }^{26}$ cognitive and linguistic functioning, ${ }^{27}$ and motor fonctions. ${ }^{28}$ The current 
COVID-19 pandemic may be considered at least as stressful as the Quebec Ice Storm and the high rates of maternal prenatal psychological distress we reported in the current study suggest that similar developmental outcomes will be observed in a substantial proportion of offspring born to women that were pregnant during the COVID-19 pandemic.

The implications for future studies are several. Our data call for biological studies including environmental, epigenetic and risk mechanisms at play in the short- and longterm consequences of prenatal psychological distress on offspring development. ${ }^{1-7}$ In this respect, future clinical research is needed to assess more thoroughly the effects of the COVID-19 pandemic on the maternal psychiatric status and offspring developmental trajectory, notwithstanding of course the outcome studies of psychosocial and pharmacological interventions in prenatal or postnatal period targeting the child and family, which is often neglected to the benefit of correlational research. ${ }^{29}$

Our study presents limitations and strengths to be considered when interpreting the findings. One strength was certainly the investigation, using the same extensive array of measurements, of two large and very similar cohorts of pregnant women from the same population, one that was assessed prior to the pandemic and the other during. A first limitation may be the difference in recruitment methods between the two cohorts, the COVID-19 cohort consisting of volunteers recruited online mainly through social media, while the pre-COVID-19 cohort was recruited in prenatal clinics. For instance, our education levels and socio-economic data suggest a more privileged sample in the COVID-19 pandemic cohort. In contrast, the two cohorts were similar in lifetime psychiatric diagnoses and age. Moreover, all comparison analyses did control for age, education level, household income, gestational age, and prior psychiatric disorder, then minimizing potential analytic biases. A second limitation of studies like this one is the impossibility of comparing the respondents to the non-respondents at enrollment, which may limit the generalizability of results. However, the level of education and the median household income of the participants were the same as those of women from Quebec's population. A third limitation in the study of such large samples is that measurements were self-reports although they were previously well tested and validated instruments.

\section{Conclusion}


In sum, pregnant women would be particularly likely to experience high levels of depression and anxiety symptoms during the COVID-19 pandemic, which may have a deleterious impact on mothers and fetuses even in socioeconomically privileged women with low-risk pregnancies. ${ }^{1,4,7}$ The present findings would call for the rapid implementation of clinical and public health strategies targeting particularly pregnant women with a history of psychiatric disorder and modest income. As recently advocated regarding the mental health impact from the COVID-19 pandemic, ${ }^{30}$ our findings may inform medical academies and decision makers i) in their recommendations to practitioners, particularly in mental health surveillance of pregnant women during the COVID-19 pandemic, such as simply inquiring about specific distress or symptoms during prenatal cares, or inquiring about the level of paternal support, ii) in their support of different forms of telehealth assistance to women with elevated psychological distress, such as toll-free helplines to answer pregnant women questions and online psychological support, iii) to encourage public health organizations to disseminate information about distress and relief of distress in pregnant women. Ideally, our findings would support a global approach of the family given that both the distressed mother and child would be in need of mental health surveillance.

Tweetable abstract: High psychological distress is observed in pregnant women during the COVID-19 pandemic @nic_berth 


\section{References}

1. Meaney MJ. Perinatal Maternal Depressive Symptoms as an Issue for Population Health. Am J Psychiatry. 2018;175(11):1084-93.

2. Bussières E-L, Tarabulsy GM, Pearson J, Tessier R, Forest J-C, Giguère Y. Maternal prenatal stress and infant birth weight and gestational age: A meta-analysis of prospective studies. Dev Rev. 2015;36:179-99.

3. Glover V. Annual Research Review: Prenatal stress and the origins of psychopathology: an evolutionary perspective. J Child Psychol Psychiatry. 2011;52(4):356-67.

4. Wu Y, Lu YC, Jacobs M, et al. Association of Prenatal Maternal Psychological Distress With Fetal Brain Growth, Metabolism, and Cortical Maturation. JAMA Netw Open. 2020;3(1):e1919940.

5. Madigan S, Oatley H, Racine N, et al. A Meta-Analysis of Maternal Prenatal Depression and Anxiety on Child Socioemotional Development. J Am Acad Child Adolesc Psychiatry. 2018;57(9):645-57 e8.

6. Tarabulsy GM, Pearson J, Vaillancourt-Morel MP, et al. Meta-analytic findings of the relation between maternal prenatal stress and anxiety and child cognitive outcome. J Dev Behav Pediatr. 2014;35(1):38-43.

7. Dean DC, Planalp EM, Wooten W, et al. Association of Prenatal Maternal Depression and Anxiety Symptoms With Infant White Matter Microstructure. JAMA Pediatr. 2018;172(10):973-81.

8. Laplante DP, Barr RG, Brunet A, et al. Stress during pregnancy affects general intellectual and language functioning in human toddlers. Pediatr Res. 2004;56(3):400-10.

9. Buekens P, Alger J, Breart G, Cafferata ML, Harville E, Tomasso G. A call for action for COVID-19 surveillance and research during pregnancy. Lancet Glob Health. 2020.

10. Thapa SB, Mainali A, Schwank SE, Acharya G. Maternal mental health in the time of the COVID-19 pandemic. Acta Obstet Gynecol Scand. 2020.

11. Gravel R, Connolly D, Bédard M. Enquête sur la Santé des les Collectivités Canadiennes (ESCC): santé mentale et bien-être, cycle 2.1 Available online at https://www.stat.gouv.qc.ca/enquetes/sante/escc.html. (Accessed May 1, 2020).

12. Kessler RC, Andrews G, Colpe LJ, et al. Short screening scales to monitor population prevalences and trends in non-specific psychological distress. Psychol Med. 2002;32(6):959-76.

13. Andrews G, Slade T. Interpreting scores on the Kessler Psychological Distress Scale (K10). Aust N Z J Public Health. 2001;25(6):494-7.

14. Spies G, Stein DJ, Roos A, et al. Validity of the Kessler 10 (K-10) in detecting DSM-IV defined mood and anxiety disorders among pregnant women. Archives Womens Ment Health. 2009;12(2):69-74.

15. Watson D, Clark LA, Tellegen A. Development and validation of brief measures of positive and negative affect: the PANAS scales. J Pers Soc Psychol. 1988;54(6):1063.

16. Gaudreau P, Sanchez X, Blondin J-P. Positive and negative affective states in a performance-related setting: Testing the factorial structure of the PANAS across two samples of French-Canadian participants. Eur J Psychol Assess. 2006;22(4):240-9. 
17. Merz EL, Malcarne VL, Roesch SC, et al. Psychometric properties of Positive and Negative Affect Schedule (PANAS) original and short forms in an African American community sample. J Affect Disord. 2013;151(3):942-9.

18. Gollan JK, Hoxha D, Getch S, Sankin L, Michon R. Affective information processing in pregnancy and postpartum with and without major depression. Psychiatry Res. 2013;206(23):206-12.

19. Ashbaugh AR, Houle-Johnson S, Herbert C, El-Hage W, Brunet A. Psychometric Validation of the English and French Versions of the Posttraumatic Stress Disorder Checklist for DSM-5 (PCL-5). PLos One. 2016;11(10):e0161645-e.

20. Weathers F, Litz B, Keane T, Palmieri P, Marx B, Schnurr P. The PTSD Checklist for DSM-5 (PCL-5) National Center for PTSD. Boston, MA, USA. 2013.

21. Berthelot N, Lemieux R, Garon-Bissonnette J, Lacharité C, Muzik M. The protective role of mentalizing: Reflective functioning as a mediator between child maltreatment, psychopathology and parental attitude in expecting parents. Child Abuse Negl. 2019;95:104065.

22. Bernstein EM, Putnam FW. Development, reliability, and validity of a dissociation scale. J Nerv Ment Dis. 1986;174(12):727-35.

23. Larøi F, Billieux J, Defeldre A-C, Ceschi G, Van der Linden M. Factorial structure and psychometric properties of the French adaptation of the Dissociative Experiences Scale (DES) in non-clinical participants. Eur Rev Appl Psychol. 2013;63(4):203-8.

24. Berthelot N, Lemieux R, Garon-Bissonnette J, Muzik M. Prenatal Attachment, Parental Confidence, and Mental Health in Expecting Parents: The Role of Childhood Trauma. J Midwifery Womens Health. 2019.

25. Cao-Lei L, Massart R, Suderman MJ, et al. DNA methylation signatures triggered by prenatal maternal stress exposure to a natural disaster: Project Ice Storm. PLoS One. 2014;9(9):e107653.

26. Paxman EJ, Boora NS, Kiss D, et al. Prenatal Maternal Stress from a Natural Disaster Alters Urinary Metabolomic Profiles in Project Ice Storm Participants. Sci Rep. 2018;8(1):12932.

27. Laplante DP, Brunet A, Schmitz N, Ciampi A, King S. Project Ice Storm: prenatal maternal stress affects cognitive and linguistic functioning in 5 1/2-year-old children. $\mathrm{J}$ Am Acad Child Adolesc Psychiatry. 2008;47(9):1063-72.

28. Cao X, Laplante DP, Brunet A, Ciampi A, King S. Prenatal maternal stress affects motor function in 5(1/2)-year-old children: project ice storm. Dev Psychobiol. 2014;56(1):117-25.

29. Berthelot N, Lemieux R, Maziade M. Shortfall of Intervention Research Over Correlational Research in Childhood Maltreatment: An Impasse to be Overcome. JAMA Pediatr. 2019.

30. Duan L, Zhu G. Psychological interventions for people affected by the COVID-19 epidemic. Lancet Psychiatry. 2020;7(4):300-2. 


\section{Supporting information content}

Table S1. Number of pregnant women with missing data for all instruments in the total sample $(\mathrm{n}=1754)$

Table S2. Comparisons of pregnant women with complete data to those with missing data on clinical and demographic characteristics.

Table S3. Comparisons of the pre-Covid-19 cohort to the COVID-19 cohort including only the pregnant women with complete data $(n=1412)$.

Table S4. Factor loadings from factor analysis with Varimax rotation of the six symptoms in the COVID-19 cohort

SDiscussion - Brief description of the Quebec Ice Storm. 
Table 1. Clinical and demographic characteristics of pregnant women from the COVID-19 and pre-COVID-19 cohorts.

\begin{tabular}{|c|c|c|c|c|}
\hline Variables & Characteristics & $\begin{array}{l}\text { COVID-19 } \\
\begin{array}{c}\text { (n = 1258) } \\
N(\%)\end{array}\end{array}$ & $\begin{array}{l}\text { Pre COVID-19 } \\
\text { (n= 496) } \\
N(\%)\end{array}$ & Group differences \\
\hline \multirow{10}{*}{$\begin{array}{l}\text { Lifetime } \\
\text { psychiatric } \\
\text { disorder }^{\text {a }}\end{array}$} & Any lifetime diagnosis & $250(19.9)$ & $105(21.2)$ & $\chi 2(1)=0.37, p=.54$ \\
\hline & Major depressive disorder & $30(2.38)$ & $20(4.03)$ & \\
\hline & Anxiety disorder & $159(12.64)$ & $64(12.90)$ & \\
\hline & Bipolar disorder & $6(0.48)$ & $4(0.81)$ & \\
\hline & Eating disorder & $6(0.48)$ & $5(1.01)$ & \\
\hline & Psychotic disorder & $0(0.00)$ & $1(0.20)$ & \\
\hline & Personality disorder & $22(2.75)$ & $14(2.82)$ & \\
\hline & PTSD & $15(1.19)$ & $8(1.61)$ & \\
\hline & ADHD/ADD & $87(6.92)$ & $39(7.86)$ & \\
\hline & Others & $7(0.56)$ & $3(0.60)$ & \\
\hline \multirow{4}{*}{$\begin{array}{l}\text { Household } \\
\text { income (CND) }\end{array}$} & $<35000 \$$ & $87(6.90)$ & $74(15.90)$ & \multirow{4}{*}{$\chi 2(3)=58.94, p<.001$} \\
\hline & $35000 \$-64999 \$$ & $197(15.70)$ & $115(23.20)$ & \\
\hline & $65000 \$-94999 \$$ & $403(32.00)$ & $162(32.70)$ & \\
\hline & $\geq 95000 \$$ & $571(45.40)$ & $145(29.20)$ & \\
\hline \multirow[t]{5}{*}{ Education } & High school not completed & $13(1.00)$ & $26(5.20)$ & $\chi 2(4)=67.33, p<.001$ \\
\hline & High school diploma & $74(5.90)$ & $39(7.90)$ & \\
\hline & Collegial/professional & $490(39.00)$ & $252(50.80)$ & \\
\hline & Undergraduate degree & $401(31.90)$ & $115(23.20)$ & \\
\hline & Graduate degree & $280(22.30)$ & $64(12.90)$ & \\
\hline & & Mean (SD) & Mean (SD) & \\
\hline \multicolumn{2}{|c|}{ Maternal age (years) } & $29.35(4.04)$ & $29.07(4.65)$ & $t^{\prime}(807.36)=-1.22, p=.22$ \\
\hline \multicolumn{2}{|c|}{ Gestational age (weeks) } & $24.38(9.26)$ & $25.85(9.73)$ & $t^{\prime}(867.92)=2.88, p=.004$ \\
\hline
\end{tabular}

a Participants reported on all medical diagnoses they received according to a list of 8 diagnoses

Abbreviations. PTSD, Post-traumatic stress disorder; ADHD/ADD, Attention-deficit hyperactivity disorder / Attention deficit disorder. CND, Canadian dollar. 
Table 2. Analyses of covariance comparing the pre-Covid-19 cohort to the COVID-19 cohort of pregnant women on six psychiatric symptoms.

\begin{tabular}{|c|c|c|c|c|c|c|c|}
\hline Dependent variables & Group & $M^{1}$ & $S E$ & $d f$ & $\boldsymbol{F}$ & $\begin{array}{c}P \text { - } \\
\text { value }\end{array}$ & $\begin{array}{l}\text { Effect } \\
\text { size }\end{array}$ \\
\hline \multirow{2}{*}{$\begin{array}{l}\text { Anxiety/depression } \\
(n=1741)\end{array}$} & Pre-COVID-19 & -0.39 & 0.04 & \multirow{2}{*}{1,1734} & \multirow{2}{*}{112.93} & \multirow{2}{*}{$<.001$} & \multirow{2}{*}{0.57} \\
\hline & COVID-19 & 0.15 & 0.03 & & & & \\
\hline \multirow{2}{*}{$\begin{array}{l}\text { Negative affectivity } \\
(n=1730)\end{array}$} & Pre-COVID-19 & -0.64 & 0.04 & \multirow{2}{*}{1,1723} & \multirow{2}{*}{321.80} & \multirow{2}{*}{$<.001$} & \multirow{2}{*}{0.96} \\
\hline & COVID-19 & 0.25 & 0.03 & & & & \\
\hline \multirow{2}{*}{$\begin{array}{l}\text { Low positive affectivity } \\
(\mathrm{n}=1730)\end{array}$} & Pre-COVID-19 & -0.64 & 0.04 & \multirow{2}{*}{1,1723} & \multirow{2}{*}{316.96} & \multirow{2}{*}{$<.001$} & \multirow{2}{*}{0.95} \\
\hline & COVID-19 & 0.25 & 0.03 & & & & \\
\hline \multirow{2}{*}{$\begin{array}{l}\text { Post-traumatic stress } \\
(\mathrm{n}=1568)\end{array}$} & Pre-COVID-19 & -0.12 & 0.04 & \multirow{2}{*}{1,1561} & \multirow{2}{*}{11.40} & \multirow{2}{*}{.001} & \multirow{2}{*}{0.19} \\
\hline & COVID-19 & 0.06 & 0.03 & & & & \\
\hline \multirow{2}{*}{$\begin{array}{l}\text { Dissociation } \\
\text { Absorption } \\
(n=1468)\end{array}$} & Pre-COVID-19 & -0.15 & 0.04 & \multirow{2}{*}{1,1461} & \multirow{2}{*}{16.32} & \multirow{2}{*}{$<.001$} & \multirow{2}{*}{0.22} \\
\hline & COVID-19 & 0.07 & 0.03 & & & & \\
\hline \multirow{2}{*}{$\begin{array}{l}\text { Dissociation/ } \\
\text { Depersonalization } \\
(\mathrm{n}=1462)\end{array}$} & Pre-COVID-19 & -0.17 & 0.05 & \multirow{2}{*}{1,1455} & \multirow{2}{*}{19.71} & \multirow[b]{2}{*}{$<.001$} & \multirow[b]{2}{*}{0.25} \\
\hline & COVID-19 & 0.08 & 0.03 & & & & \\
\hline
\end{tabular}

ANCOVAs were performed to examine group differences on the psychiatric symptoms separately. Scores were previously converted to z scores. The analyses controlled for age, gestational age, education level, household income and lifetime psychiatric disorders. Effect sizes (ES) were calculated using the difference of adjusted means between participants of the COVID-19 and pre-COVID-19 cohorts divided by a pooled standard deviation.

Abbreviations. $M$, Estimated marginal means; SE, Standard error; df, degree of freedom; $P$, probability value; ES, Effect sizes. 
Table 3. Multiple regressions assessing the association between sociodemographic characteristics and the three clusters of symptoms in pregnant women of the COVID-19 cohort.

\begin{tabular}{|c|c|c|c|c|c|c|c|c|}
\hline Criteria & Predictors & $\begin{array}{c}\text { Adjusted } \\
\mathbf{R}^{2}\end{array}$ & B & SE & $\boldsymbol{\beta}$ & P-value & $\begin{array}{l}95 \% \mathrm{Cl} \\
\text { lower } \\
\text { bound }\end{array}$ & $\begin{array}{l}95 \% \mathrm{Cl} \\
\text { upper } \\
\text { bound }\end{array}$ \\
\hline \multirow{5}{*}{$\begin{array}{l}\text { Mood and } \\
\text { anxiety } \\
\text { symptoms }\end{array}$} & Lifetime psychiatric disorder & \multirow{5}{*}{0.07} & 0.52 & 0.07 & 0.21 & $<.001$ & 0.39 & 0.64 \\
\hline & Age & & -0.01 & 0.006 & -0.03 & .24 & -0.02 & 0.01 \\
\hline & Education & & -0.08 & 0.03 & -0.07 & .02 & -0.14 & -0.02 \\
\hline & Household income & & -0.03 & 0.01 & -0.08 & .005 & -0.06 & -0.01 \\
\hline & Gestational age & & 0.001 & 0.003 & 0.009 & .72 & -0.004 & 0.01 \\
\hline \multirow{5}{*}{$\begin{array}{l}\text { Dissociative } \\
\text { symptoms }\end{array}$} & Lifetime psychiatric disorder & \multirow{5}{*}{0.06} & 0.34 & 0.07 & 0.14 & $<.001$ & 0.21 & 0.47 \\
\hline & Age & & -0.001 & 0.007 & -0.01 & .85 & -0.01 & 0.01 \\
\hline & Education & & -0.07 & 0.03 & -0.07 & .03 & -0.13 & -0.006 \\
\hline & Household income & & -0.06 & 0.01 & -0.14 & $<.001$ & -0.08 & -0.03 \\
\hline & Gestational age & & 0.00 & 0.003 & 0.001 & .96 & -0.01 & 0.01 \\
\hline \multirow{5}{*}{$\begin{array}{l}\text { Low positive } \\
\text { affectivity }\end{array}$} & Lifetime psychiatric disorder & \multirow{5}{*}{0.03} & 0.20 & 0.07 & 0.08 & .003 & 0.07 & 0.33 \\
\hline & Age & & -0.08 & 0.007 & -0.03 & .22 & -0.02 & 0.01 \\
\hline & Education & & 0.12 & 0.03 & 0.12 & $<.001$ & 0.06 & 0.19 \\
\hline & Household income & & 0.02 & 0.01 & 0.05 & 0.14 & -0.01 & 0.04 \\
\hline & Gestational age & & -0.01 & 0.003 & -0.09 & $<.001$ & -0.02 & -0.004 \\
\hline
\end{tabular}

A factor analysis was performed on the COVID-19 cohort to regroup the six clinical symptoms into a limited number of meaningful clusters. The analysis yielded three domains of symptoms: mood and anxiety symptoms (including symptoms of anxiety/depression, negative affectivity and symptoms of PTSD), dissociative symptoms and low positive affectivity (see Table S4). Three multiple regressions were next performed with the COVID-19 women's clinical and sociodemographic characteristics as predictors and the factor scores as criteria. The significance level was fixed at $p<.02$.

Abbreviations. B, understandardized beta; $\beta$, standardized beta; $S E$, Standard error for the understandardized beta; $P$, probability value; $\mathrm{Cl}$, confidence intervals. 